\title{
Marcel Proust oder die Reflexionen des Lesens
}

Marcel Proust hat die Entwicklung Joris-Karl Huysmans', die mit $A$ rebours in eine sehr fruchtbare, aber gleichwohl dauerhafte Aporie eingetreten war, im Kontext der literargeschichtlichen Entwicklung des Romans in Frankreich aufmerksam beobachtet und wieder aus der Sackgasse herausgeholt. Er hat damit in gewisser Weise die ,Literaturen am Ende‘ aus ihrer finisekulären Ausweglosigkeit befreit und der französischen wie europäischen Romangeschichte neue Horizonte beschert.

Marcel Proust nimmt eine Vielzahl literarisch-kultureller Elemente Huysmans' auf. Er wird in seiner eigenen Persönlichkeit und Rolle als Dandy eine Vielzahl von Bestandteilen der literarischen Vorlage des Esseintes in eigene Lebenspraxis verwandeln und die Faubourgs der Aristokratie, ganz wie der junge Huysmans, snobistisch und geradezu szientifisch beäugen. Proust wird sich mit der Kunst aus der Perspektive des Dilettanten beschäftigen, Paris auf den Spuren Baudelaires als Flaneur durchstreifen, als Liebhaber und Amateur der Künste auftreten.

Er präsentiert dabei eine ungeheure Verdichtung von Traditionen und Verfahren, die sich in seinem eigenen Leben wie in seiner Kunst kreuzten. Das Thema der Schwäche und vor allem der Krankheit wird er in seinem eigenen Leben, aber auch im Leben seiner fiktionalen Gestalten in eine direkte Beziehung zur Erzeugung von Kunst stellen - und dies in so starkem Maße, dass wir ihn nicht nur als einen der Überwinder des fin de siècle, sondern zugleich als einen der großen Repräsentanten des Nachdenkens über die Grundlagen der Literatur und insbesondere des Lesens begreifen dürfen. Denn Proust ist nicht nur der ,Erfinder einer ausgefeilten Erinnerungskunst, nicht nur der sorgfältige Beobachter der gesellschaftlichen Veränderungen, welche Frankreich am Übergang zum 20. Jahrhundert charakterisieren, sondern auch Vermittler des 19. mit einem neuen Jahrhundert, dem er die weiten Horizonte literaturtheoretischer Reflexion eröffnete. Kaum ein Theoretiker dieses neuen Jahrhunderts, der sich nicht in irgendeiner Form auf Proust bezöge. Sein Romanzyklus A la recherche du temps perdu wird während eines langen 20. Jahrhunderts den immer wieder gesuchten Referenzpunkt für die unterschiedlichsten Theorieansätze ebenso der ancienne wie der nouvelle critique, ebenso der Konstanzer Rezeptionsästhetik wie der französischen Neoavantgarden bilden. Im Mittelpunkt dieser letztgenannten Ansätze steht das Lesen.

Da wir im Rahmen unserer Vorlesung leider nicht in eine längere Debatte über Marcel Proust eintreten können - dies bleibt einer anderen Vorlesung vorbehalten -, müssen wir etwas Zeit sparen und auf eine Kurzbiographie des 1871 
geborenen französischen Romanciers verzichten. Bei vielen Autorinnen und Autoren des ausgehenden 19. Jahrhunderts lässt sich beiderseits des Atlantik gut beobachten, welch enorme Bedeutung der Lektüre zukommt und wie sehr der Raum der Kultur zu einem Bücherraum geworden ist.

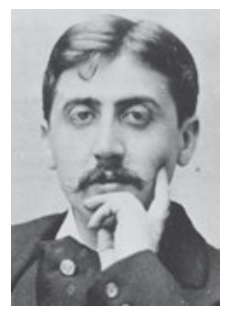

Abb. 111: Marcel Proust, fotografiert von Otto Wegener um 1895-1896.

In der Tat ist es auffällig, wie sehr die Problematik des Lesens und der Lektüre ebenso im Roman wie im Essay thematisiert wurde, wie sehr die Bedeutung des Lesens in den unterschiedlichsten Gebieten - ebenso im Bildungsbereich wie in der Frage der Bildungsvermittlung, im Bereich der Kunst wie in der Literatur - intensiv behandelt wurde. Auch bei Marcel Proust wird diese Frage eine entscheidende Stellung einnehmen, wird sie es ihm doch erlauben, der Konversation, die unverantwortlich und mondän ist, die tiefere Auseinandersetzung entgegenzustellen, für welche die konzentrierte und selbstreflexive Lektüre einsteht.

Damit aber wird Proust - und darauf hat bereits der französische Theoretiker Antoine Compagnon hingewiesen - einen Gegensatz einführen, den er dann 1908 in seinem Contre Sainte-Beuve ins Zentrum rücken wird: jenen Gegensatz, der das moi social vom moi créateur trennt und damit schon auf dieser Ebene eine Art Autonomisierung des Künstlers (und damit a fortiori auch der Kunst) ins Blickfeld rückt und weiter vorantreibt. Das bedeutet nicht, dass Proust sich nicht für die Welt, für die Gesellschaft interessiert hätte. Ganz im Gegenteil gibt es zu seiner Zeit keinen aufmerksameren, präziseren Beobachter all jener gesellschaftlichen Transformationen und Umbrüche, welche die Zeit vor Ausbruch des Ersten Weltkrieges prägen und letztlich die Alte Welt im Wirbel der Grande Guerre untergehen lassen. Auch er wird, ganz wie Anatole France, die Erklärung der Intellektuellen zugunsten von Dreyfus unterschreiben und damit in der sogenannten Dreyfus-Affäre ${ }^{1}$ politische Position beziehen. Und doch geht es

1 Vgl. hierzu Jurt, Joseph: Politisches Handeln und ästhetische Transposition: Proust und die Dreyfus-Affäre. In: Maas, Edgar / Roloff, Volker (Hg.): Marcel Proust. Lesen und Schreiben. Frankfurt am Main: Insel Verlag 1983, S. 85-107; sowie ders.: Au nom de la vérité et de la justice. Les valeurs littéraires dans le champ politique au moment de l'Affaire Dreyfus. In: Baudelle, Yves / 
ihm letztlich um eine Kultur und Literatur, deren Sitz im Leben von einer großen Autonomie, einem großen Freiraum für das Ästhetische geprägt ist.

Marcel Proust hatte sich als Kunstliebhaber bereits Ende der neunziger Jahre des 19. Jahrhunderts mit dem britischen Kunstkritiker und Gesellschaftsreformer John Ruskin intensiv auseinandergesetzt. Es sei an dieser Stelle nur am Rande angemerkt, dass Ruskin für keinen Geringeren als Gabriele d’Annunzio eine nicht unwichtige Bezugsfigur geworden war. Der italienische Romancier hatte erkannt, wie bedeutend die von Ruskin in den Vordergrund gerückten englischen präraffaelitischen Maler waren und wieviel wichtiger noch für ihn jene Ästhetik der italienischen Präraffaeliten sein könnte, die vor Raffaello gemalt hatten und die nun in den Kunstkonzeptionen des fin de siècle eine herausragende Rolle spielen sollte. Erlaubte John Ruskin D’Annunzio den über England legitimierten Rückgriff auf die italienische Malerei vor Raffaello, so erlaubte es Ruskin Marcel Proust, seine Ästhetik in jenem Jahrzehnt voranzutreiben, das dem der Niederschrift von $A$ la recherche du temps perdu vorausging. Ruskin wird stets ein wichtiger Bezugspunkt für Prousts ästhetische Konzeptionen bleiben.

Der Verfasser der Recherche hatte bereits Ruskins La Bible d'Amiens übersetzt zu einem Zeitpunkt, als durch Europa und vor allem aber Frankreich, eine wahre Welle der Beschäftigung mit dem im Jahre 1900 verstorbenen schottischen Kunstkritiker einsetzte. Proust wird 1909 Ruskin auch eines seiner Pastiches widmen, das er freilich zu Lebzeiten nicht mehr veröffentlichen sollte; eines jener Pastiches, mit Hilfe derer es Proust gelang, den prägenden Einfluss anderer Schriftsteller loszuwerden und eigene Formen und Strategien des Schreibens wie des Lesens zu entwickeln. Bald schon sollte Marcel Proust seinen gigantischen Romanentwurf Jean Santeuil hinter sich lassen und mit den ersten Arbeiten an A la recherche du temps perdu beginnen.

Bereits 1865 war Ruskins Sesame and Lilies in London erschienen, ein Buch, das neben einem Vorwort zwei Vorträge Ruskins enthielt, in dessen erstem sich der schottische Kunstkritiker zu Notwendigkeit und Bedeutung des Lesens äußerte. Dabei ging es ihm in einem Vortrag im Rahmen einer Veranstaltung, bei der Geld für die Eröffnung einer öffentlichen Bücherei gesammelt werden sollte, um die soziale Dimension des Lesens und die gesellschaftliche Wichtigkeit einer auch den Frauen zugänglichen Bildung.

Deguy, Jacques / Morzevski, Christian (Hg.): A la recherche d'un sens. Littérature et vérité. Mélanges offerts à Monique Gosselin-Noat. Lille: Presses Universitaires du Septentrion 2004, S. 347-359. 


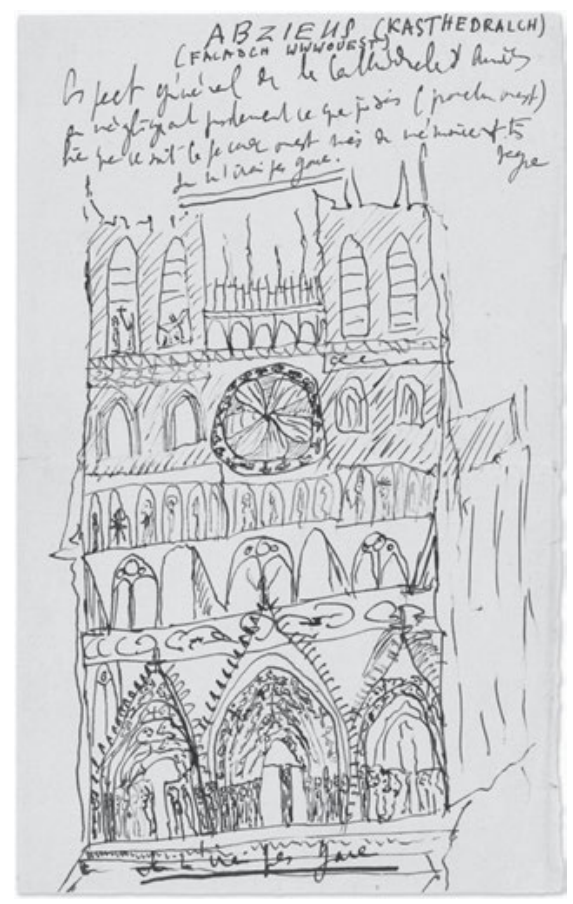

Abb. 112: „Abziens (kasthedralch)“: Von Marcel Proust handschriftliche gezeichnete Skizze der Kathedrale von Amiens, circa 1901-1904.

Für Marcel Proust freilich stellte sich die Frage der Lektüre durchaus anders, nämlich als jene Form der Beschäftigung und Wissensaufnahme, in der das Individuum in einsamer, versunkener, von den anderen abgetrennter Lektüre gleichsam zu sich selbst und seinem tieferen, ja zu seinem wahren Ich findet. Diese Vorstellungen hat er dem Vorwort seiner 1905 erschienen Übersetzung von Sésame et les Lys unter dem Titel Sur la lecture (auch Journées de lecture) anvertraut, einem Text, der uns tief in die Ästhetik des französischen Autors einführt. Sehen wir uns den Beginn dieses außergewöhnlich schönen, wegweisenden Vorworts einmal näher an:

Es gibt vielleicht keine Tage unserer Kindheit, die wir so vollständig erlebt haben wie jene, die wir glaubten verstreichen zu lassen, ohne sie zu erleben, jene nämlich, die wir mit einem Lieblingsbuch verbracht haben. Alles, was sie, wie es schien, für die anderen erfüllte und was wir wie eine vulgäre Unterbrechung eines göttlichen Vergnügens beiseiteschoben: das Spiel, zu dem uns ein Freund bei der interessantesten Stelle abholen wollte; die störende Biene oder der lästige Sonnenstrahl, die uns zwangen, den Blick von der Seite zu heben oder den Platz zu wechseln; die für die Nachmittagsmahlzeit mitgegebenen Vorräte, die wir unberührt neben uns auf der Bank liegen ließen, während über unserem Haupt die Sonne am blauen Himmel unaufhaltsam schwächer wurde; das Abendessen, zu dem wir zurück ins Haus mussten und währenddessen wir nur daran dachten, sogleich danach in unser 


\begin{abstract}
Zimmer hinaufzugehen, um das unterbrochene Kapitel zu beenden, all das, worin unser Lesen uns nur Belästigung hätte sehen lassen müssen, grub im Gegenteil eine so sanfte Erinnerung in uns ein (die nach unserem heutigen Urteil um so vieles kostbarer ist als das, was wir damals mit Hingabe lasen), dass, wenn wir heute manchmal in diesen Büchern von einst blättern, sie nur noch wie die einzigen aufbewahrten Kalender der entflohenen Tage sind, und es mit der Hoffnung geschieht, auf ihren Seiten die nicht mehr existierenden Wohnstätten und Teiche sich widerspiegeln zu sehen. ${ }^{2}$
\end{abstract}

Dieser wahrhaft Proust'sche Satz, nach dem wir erst einmal tief durchatmen müssen, hat es wahrhaft in sich! Dies ist zum einen schon deshalb der Fall, weil er eine so komplexe hypotaktische Struktur aufbaut, wie sie nicht nur für Prousts gesamtes Schreiben, sondern auch für eine ästhetizistische Schreibtradition typisch ist, für Joris-Karl Huysmans' A rebours wie für viele nachfolgende Autorinnen und Autoren des fin de siècle, sondern weil sie auch das Proust'sche Grundthema schlechthin vorstellt, den Rückblick auf das für immer Entschwundene, auf die für immer unzugängliche Kindheit. Auf diese kunstvolle Weise wird das Schreiben und mehr noch das Lesen an die Erinnerung geknüpft, wird all das, was unseren gegenwartsbezogenen Sinnen abhandengekommen ist, was ihrer Wahrnehmung für immer verwehrt zu sein schien, ja was nicht einmal mehr in der Wirklichkeit existieren mag, wieder ins Leben zurückgeholt. Mit einem Wort: Die vergangene Zeit, die verlorene Zeit, wird im Kalenderspiel dieser Seiten eines Lieblingsbuches wieder unmittelbar lebendig, greifbar und sinnlich begreifbar.

Denn das Lesen wird in der obigen, fast atemlosen Passage als die Lektüre in der Kindheit vorgestellt, eine Thematik, die vielfach in Prousts Werk zurückkehren wird, die aber erst sehr spät dann zu jener Proust'schen Wendung in der Recherche führen sollte, in welcher die Geschichte einer vocation, einer Berufung zum Schreiben, zum Roman selbst werden wird - so jedenfalls eine der für mich überzeugendsten Deutungslinien von Prousts A la recherche du temps perdu. Hier ist freilich noch die dritte Person, ebenso im Singular wie im Plural, Garantin der direkten Übertragung vom Erzähler zum Lesepublikum, die Proust in der Recherche - im Gegensatz zu seinem frühen, großartig gescheiterten Romanprojekt Jean Santeuil dann der ersten Person Singular anvertrauen und übertragen wird. Prousts Überlegungen in Sur la lecture sind freilich wesentlich mehr als eine Fingerübung für den großen Romanzyklus, den er kurze Zeit später dann gegen die vorrückende, sein Leben beendende Zeit in einem Zimmer mit dichten Korkwänden - ein wenig so wie sein jüngerer Zeitgenosse Albert Einstein in seinem

2 Proust, Marcel: Sur la lecture. Arles: Actes Sud 1993, S. 9 f. 
,Häusle‘ unweit von hier in Caputh - gegen alle störenden Geräusche abschließen sollte.

Gleich die ersten Worte der obigen Passage weisen auf einen vermeintlichen Gegensatz hin: den Gegensatz zwischen Lesen und Leben, mit dem wir uns bereits $\mathrm{zu}$ Beginn unserer Vorlesung beschäftigt haben. In seinen hintergründigen Überlegungen macht Proust mit Blick auf die Kindheit das Lesen gerade $\mathrm{zu}$ jenen Augenblicken und Zeiträumen, die wir am intensivsten, am vollkommensten gelebt und - um erneut den Dilthey'schen Begriff zu verwenden - durcherlebt haben. Die vielen kleinen, unscheinbaren Details, die in der Folge in Erscheinung treten, bilden eine Art effet de réel, ${ }^{3}$ welcher das Erleben der Realität noch übersteigert, ja überhöht. Die Wirklichkeit, das sind diese unzähligen Momente und Elemente, ein störender Lichtstrahl, eine summende Biene, die stur einzuhaltenden Essenszeiten. Sie alle sind im Hegel'schen Sinne aufgehoben in der Lektüre. In jedem Falle aber enthält hier das Lebenswissen, das sich mit der Lektüre in der Kindheit verbindet, die Dimension eines sich Erinnerns an ein vollkommen gelebtes Leben, das nur als vollkommenes Lesen denkbar zu sein scheint und allein in seiner Gesamtheit, also vollständig, vorgestellt werden kann. Das Buch tritt an die Stelle der Welt, wird zu dieser Welt, in der wir längst zu leben begonnen haben. Das Lesen erscheint als verdichtete Form des Lebens.

Doch da ist noch etwas, das uns an den Beginn unserer Vorlesung gemahnt. Denn die Anfangssituation ist erstaunlicherweise mit Italo Calvinos Romanincipit von Se una notte d'inverno un viaggiatore in einen unmittelbaren Zusammenhang zu bringen, wird hier doch die Situation derjenigen oder desjenigen, der das Buch öffnet, in gewisser Weise gespiegelt, geht es doch um die Erfahrung einer Lektüre und der notwendigen Schaffung von Einsamkeit, der Herstellung eines ungestörten Raumes, den Lektüre als einsame Tätigkeit nun einmal benötigt.

Damit aber wird wie bei Italo Calvino die Lektüre zum einen gegen andere Vergnügungen gestellt, etwa gegen den Freund, der den kleinen Marcel zum Spielen abholen möchte, oder gegen die Eltern, die ihren Sohn zum Essen herbeiholen wollten; und zum anderen gegen die Konversation, letztlich das Gegenteil der Innerlichkeit und Selbstfindung in der Tiefe, jene Oberfläche, die sich lärmend vor das Erkennen des Eigentlichen in der Tiefe stellt. Die Analogien zu

3 Vgl. Barthes, Roland: L'effet de réel. In (ders.): Le bruissement de la langue. Essais critiques IV. Paris: Seuil 1984, S. 167-174 (ursprünglich 1968 in der Zeitschrift Communications erschienen). 
Se una notte d'inverno un viaggiatore sind offenkundig und könnten weiter durchgespielt werden.

Ähnlich wird die Erzählerfigur im letzten Band von Marcel Prousts A la recherche du temps perdu betonen, der Schriftsteller solle seinen Roman nicht außerhalb seiner selbst suchen und erfinden, sondern ihn in sich selbst suchen und finden; denn wir alle tragen unseren Roman sozusagen in uns - die wenigsten aber lassen ihn zur Sprache kommen. Und ich müsste eigentlich sagen: zur Schrift kommen. Denn genau um diesen Übergang geht es!

Wollen wir diese Raumstrukturen also noch einmal aufeinander beziehen, so könnten wir sagen, dass eine Metaphorik der Tiefe, man könnte auch Metaphysik der Tiefe sagen, ebenso Huysmans wie Proust oder auch Sigmund Freud gemeinsam ist. Die Wahrheit liegt im Innern unseres Selbst, wo auch der späte Huysmans sie suchte, und nicht in den Oberflächenbeschreibungen eines Zola'schen Romanmodells. Welche Funktion hat aber dann die Lektüre, ist es doch die Stimme eines Anderen, die sich zu Wort meldet?

Marcel Proust grenzt sich an dieser Stelle deutlich von René Descartes ab, der die Lektüre wichtiger Bücher als Gespräche mit den herausragenden Männern der Vergangenheit verstand. Für Proust ist die Lektüre gerade nicht mit einer Konversation, einem eher oberflächlichen Gespräch, vergleichbar:

Ich habe in den Anmerkungen, welche ich diesem Band beigefügt habe, zu zeigen gesucht, dass die Lektüre nicht einfach mit einer Konversation gleichgestellt werden kann, und fände sie mit dem weisesten der Menschen statt; denn der wesentliche Unterschied zwischen einem Buch und einem Freund besteht nicht in ihrer jeweils größeren oder geringeren Weisheit, sondern in der Art und Weise, wie man mit beiden kommuniziert, beruht doch die Lektüre, ganz im Gegensatz zur Konversation, für einen jeden von uns darauf, die Mitteilung eines anderen Denkens zu empfangen, wobei wir allerdings alleine bleiben, das heißt weiterhin jene geistige Macht genießen, welche man in der Einsamkeit besitzt, während die Konversation unmittelbar zerstreuend wirkt, auch wenn man weiterhin inspiriert bleiben kann und die fruchtbare Arbeit des Geistes an sich selbst erhalten bleibt. ${ }^{4}$

Entscheidend ist in dieser Passage wie in Sur la lecture überhaupt, dass nicht nur der Akt des Schreibens, sondern gerade auch der Akt des Lesens überdacht und als geistig-kreativer Kommunikationsakt verstanden wird. Damit ist das Schreiben Prousts in gewisser Weise in die Aktivität des Lesens integriert, so wie Proust auch bei einigen Schriftstellern oder Philosophen wie Ralph Waldo Emerson betont, dass diese kaum einmal mit dem Schreiben anfingen, ohne zuvor einige Seiten großer Autoren gelesen $\mathrm{zu}$ haben. Lesen und Schreiben, Rezeption

4 Proust, Marcel: Sur la lecture, S. 29. 
und Produktion werden hier aufeinander bezogen und füreinander fruchtbar gemacht, stehen aber beide in einem schroffen Gegensatz zur Konversation, zum Austausch von Freundlichkeiten und Unverbindlichem, dem keinerlei Dauer und geistige Macht, puissance intellectuelle, beschieden ist.

Ist das Buch und dessen Lektüre auf einem Akt der Freundschaft, der amitié aufgebaut, so ist die Konversation auf dem Prinzip der amabilité, dem Austausch von Freundlichkeiten, errichtet. Besonders aufschlussreich scheint, dass somit Prousts eigenes Schreiben gleichsam auf einer Poetik und zugleich Legetik errichtet ist, also einer Ästhetik des Lesens, die grundlegende Regeln für ein Verständnis von Lektüreprozessen entwickelt hat. Insofern führt uns auch die Legetik Prousts, wie sie in Sur la lecture entfaltet wird, direkt ins Zentrum des Proust'schen Denkens und mehr noch seines Schreibens. Lesen ist der Schlüssel zur kreativen Potenz des Menschen.

Nähern wir uns aber einer anderen, einer weiteren Dimension der Lektüre in Marcel Prousts Ästhetik:

\begin{abstract}
Solange die Lektüre für uns die Initiatorin ist, deren magische Schlüssel uns tief in unserem Innern die Türen der Gemächer öffnen, in die wir nicht einzudringen wussten, so ist ihre Rolle in unserem Leben heilbringend. Sie wird hingegen gefährlich, wenn die Lektüre, anstatt uns zum persönlichen Leben des Geistes zu erwecken, dazu tendiert, an dessen Stelle zu treten, wenn uns die Wahrheit nicht länger als ein Ideal, welches wir allein durch den intimen Fortschritt unseres Denkens und durch die Anstrengungen unseres Herzens verwirklichen können, sondern als ein materielles Ding erscheint, das zwischen den Seiten und Blättern der Bücher aufbewahrt wird wie ein Honig, der von anderen hergestellt wäre und bei dem wir uns nur die Mühe machen müssten, ihn von den Regalbrettern der Bibliotheken herunterzuholen, um ihn anschließend, Körper und Geist in vollkommener Ruhe, passiv zu kosten. ${ }^{5}$
\end{abstract}

Marcel Proust stellt für seine Leser an dieser Stelle ein Warnschild auf: Er zeigt die Grenzen der Lektüre und ihre Gefahren, insofern sie das Leben nicht mehr bereichert und ihm eine zusätzliche Tiefe, die Schlüssel zu etwas für uns sonst Unerreichbarem gibt, sondern sich dem Leben substituiert, also an die Stelle des Lebens in seiner persönlichen Entfaltung tritt. Dann wird Lektüre zu einer negativen, ja zerstörerischen, die Entfaltung unseres Geistes behindernden Kraft und Tätigkeit. Sie gerät zu einem zwar lustvollen, aber insgesamt doch rein passiven und unkreativen Honigschlecken.

Wenn uns das Lesen also nicht zum eigenen geistigen Leben erweckt, also zu jenem Impuls wird, der uns zum eigenen Schaffen anregt, sondern konsumiert

5 Proust, Marcel: Sur la lecture, S. $37 \mathrm{f}$. 
wird wie ein fertig dargebotener Honig, dann wird es zur Gefahr für die Ausbildung einer kreativen Persönlichkeit. Prousts Forderung ist folglich die nach einem aktiven, die eigenen Geisteskräfte ständig weiterentwickelnden Lesepublikum. Zugleich dient sie als Warnung vor einer passiven Lektüre, die das fertig Dargebotene nur einzuheimsen sucht, ohne dabei auf größere Anstrengungen angewiesen zu sein. Die Leserschaft ist damit aufgerufen, durch Lektüre ständig an ihrer eigenen Bildung weiterzuarbeiten: Lesen darf nicht zum Konsum werden, sondern zu einer Vertiefung der eigenen Existenz, des eigenen Lebens! Es geht folglich um die Fähigkeit, in uns unseren eigenen Geist zu erwecken.

Dies betrifft gerade auch das Erleben unterschiedlicher Ebenen der Zeit, also dessen, was wir die Gleichzeitigkeit des Ungleichzeitigen nennen können. Am Ende seines Textes spricht Proust von der Lektüre der göttlichen Commedia des Dante Alighieri, die plötzlich eine andere Zeit, eine eigentlich längst vergangene Zeit, in unsere Gegenwart einführt und gleichsam unserem Erleben (beziehungsweise Nacherleben) darbietet.

In diesen Wendungen erscheint bereits jene zentrale Idee, die Marcel Proust in A la recherche du temps perdu ästhetisch eindrucksvoll entfalten wird, in nuce als die Möglichkeit der Literatur und der Lektüre, die Gegenwart geradezu traumartig mit anderen Zeitebenen zu durchsetzen und diese Multitemporalität für uns zugänglich zu machen. Dieses un peu de temps à l'état pur, die Zeit im Reinzustand, wird durch Lektüre erreichbar, solange sie eine bewusste, aktive und zugleich doch dem Tagträumen nahestehende Aktivität ist, wie wir dies im Folgenden im Nachklang der Dante-Lektüre ganz am Ende von Sur la lecture erfahren. Hören wir noch einmal die Stimme Prousts zum höchst kreativen Vorgang des Lesens und zur Verschiedenartigkeit der Zeiten in einem literarischen Text:

Darum herum zirkulieren die aktuellen Tage, jene Tage, welche wir leben, pressen sich summend um die Säulen, aber dann halten sie plötzlich inne, fliehen wie verjagte Bienen; denn sie sind nicht in der Gegenwart, diese hohen und feinen Enklaven der Vergangenheit, sondern in einer anderen Zeit, zu welcher der Gegenwart der Zutritt untersagt ist. Um die rosafarbenen Säulen, zu ihren breiten Kapitelen hochragend, pressen sich und summen die aktuellen Tage. Doch sich zwischen sie drängend, schieben die Säulen sie beiseite, wobei sie mit ihrer ganzen zarten Dichte den unberührbaren Platz der Vergangenheit reservieren: einer Vergangenheit, die ganz familiär inmitten der Gegenwart aufgetaucht ist mit dieser ein wenig irrealen Farbe der Dinge, welche sie uns in einer Art Illusion nur wenige Schritte entfernt sehen lässt, obwohl sie doch in Wirklichkeit viele Jahrhunderte entfernt sind; sich in ihrem ganzen äußeren Bilde ein wenig zu direkt dem Geiste präsentierend, ihn ein wenig bestaunend, wie man es mit einem Wiedergänger aus einer längst untergegangenen Zeit machte; und dennoch einfach da, in unserer Mitte, nahe, Seite an Seite, anfassbar, unbeweglich, im Lichte der Sonne. ${ }^{6}$

6 Proust, Marcel: Sur la lecture, S. 53f. 
Die ganze architektonische Metaphorik Marcel Prousts, dessen Recherche nur zu oft aus gutem Grund mit einer Kathedrale der Zeit verglichen wurde, ist in diesem letzten Zitat aus Sur la lecture wunderbar ausgeprägt, klar und doch verwirrend unscharf. Es kommt in dieser Passage durch die Lektüre also gerade nicht zur Vermischung und Überlagerung der Zeiten, sondern zu einem ganz anderen Phänomen, gleichsam der Errichtung einer eigenen, diskontinuierlichen und aus der Kontinuität der Zeiten gerissenen Zeitinsel, wie sie hier - die Szenerie spielt nicht ganz zufällig in Venedig - gemäß des genius loci anhand einer Säulenarchitektur vorgeführt wird. Kein Wunder, dass gerade in der von Inseln gebildeten und doch als Einheit wahrgenommenen Stadt Venedig Marcel Proust eine Zeitvorstellung entwickeln konnte, welche zutiefst insulären, ja archipelischen Zuschnitts ist und Zeit als diskontinuierliche Einheit vor Augen führt. Den Canale Grande innerhalb dieser Gleichzeitigkeit des Ungleichzeitigen verlor der französische Autor gleichwohl nicht aus seinen Augen.

Die Gegenwart ist nicht in der Lage, diese Enklaven einer anderen, distanten Zeit $\mathrm{zu}$ überwältigen und in sich einfach zum Verschwinden zu bringen. Vergangenheit ist in Gegenwart eingelagert, ja mehr noch: sie ist greifbar, anschaulich, lebendig. Die Zeit findet ihren eigenen, nicht $\mathrm{zu}$ verletzenden und nicht zu überwältigenden Ort der Vergangenheit, und zwar inmitten der Gegenwart. Die Zeit ist plötzlich aufgespalten, gibt andere Zeit-Räume frei, die uns so nah und doch gleichsam irreal anmuten. Und all dies lässt die Lektüre entstehen!

Die Zeitlichkeit der Lektüre wird in diesen Wendungen deutlich markiert; also nicht nur eine eigene Räumlichkeit des Lesens, wie wir sie von Beginn unserer Vorlesung an genau sich abzeichnen sahen in den Orten des Lesens, den Bibliotheken, den Betten, den Sitzgelegenheiten, die der Lektüre dienen, sondern in der Errichtung eigener ,Räume‘ der Zeit, eigener ,Zeit-Räume‘, die sich nicht ineinander auflösen, sondern nebeneinander, ja sogar ineinander fortbestehen. Lesen wird somit $\mathrm{zu}$ einem transtemporalen Phänomen, insoweit die Lektüre verschiedenste, zum Teil Jahrhunderte voneinander entfernte Zeiten quert, ohne diese freilich miteinander verschmelzen zu lassen. Die Suche nach der verlorenen Zeit, nach der verlorenen Kindheit des Erzählers, aber auch nach den untergegangenen Jahrhunderten in den Kunstwerken und architektonischen Strukturen unserer Städte, konzentriert sich auf jene materiellen Dinge, die in den so metaphernbeladenen Passagen Marcel Prousts dennoch ganz konkrete Dingwelten entfalten. Auf der Suche nach un peu de temps à l'état pur werden dies etwa eine sich im Tee auflösende Madeleine oder die ungleichen Pflastersteine unter den Füßen der Erzählerfigur sein. Doch darauf kann ich an dieser Stelle der Vorlesung nicht weiter eingehen ... 
Man könnte analog zur Unterscheidung zwischen Erzählzeit und erzählter Zeit zwischen einer Lesezeit und einer gelesenen Zeit sprechen, die sich ebenfalls nicht einfach ineinander auflösen und miteinander mischen. Transtemporal ist diese Leseerfahrung und Zeiterfahrung - oder müssten wir hier nicht eher von einem Erleben als von einer Erfahrung sprechen? -, weil der Leser selbst zwischen diesen verschiedenen Zeit-Räumen sich hin- und her bewegt, also eine Bewegung in der Zeit vollführt, während er doch auf einem schönen Platz im Herzen von Venedig sitzt und die Stadt auf sich wirken lässt. Mit Proust gelangen wir in den Innenraum einer Lektüre, der letztlich von der Zeit gebildet wird: der Eigenzeitlichkeit des Lesens und des Gelesenen, die sich in uns selbst ausbreitet und zugleich in uns selbst verankert ist. Auch in diesem Sinne ist Literatur - anders als Malerei - eine Zeit-Kunst: und die Kunst des Lesens eine Kunst, die unterschiedlichste Zeiten durch die eigene Bewegung miteinander in Verbindung $\mathrm{zu}$ bringen und zueinander in Kontrast $\mathrm{zu}$ setzen sucht - transtemporal.

Viele der Szenen und Motive, die wir in Prousts $A$ la recherche du temps perdu finden und bewundern können, lassen sich bereits zumindest in nuce in seinen essayistischen, literatur- und kunstkritischen Schriften finden. Sur la lecture war hierfür ein gutes Beispiel. Dazu gehört bei Proust stets die Reflexion über das Lesen, die bei ihm immer eine Reflexion über das livre intérieur und damit über das eigene Schreiben ist; über die Entfaltung eines eigenen Romans, den zu verwirklichen der Erzähler der Recherche längst nicht aufgegeben hatte.

Ich möchte Ihnen gerne abschließend $\mathrm{zu}$ Proust zwei Überlegungen aus seinem bereits erwähnten Band Contre Sainte-Beuve vorführen, wobei uns zunächst eine Bibliotheksszene interessieren soll. Proust erzählt uns zunächst von der petite bibliothèque du second des Duc de Guermantes, wohin sich der Herzog immer dann flüchtet, wenn seine Frau, die Duchesse de Guermantes, mondänen Besuch erhält. Hierher lässt er sich dann auch seine Biskuits und seinen Sirup bringen. Der Duc de Guermantes verfügt in seiner Bibliothek über das Gesamtwerk von Honoré de Balzac, das er sehr kostbar und edel in vergoldetes Kalbsleder mit grünen Lederetiketten hat binden lassen. Er lässt sich seine Bewunderung für den französischen Romancier also wirklich etwas kosten - ein im Übrigen nicht unwichtiges Detail zur Einschätzung der Wirkung Balzacs im ausgehenden 19. Jahrhundert.

Ein weiteres Detail kommt hinzu: Der Duc de Guermantes kann praktisch alle Romane Balzacs auswendig und kennt sämtliche - und es sind nicht wenige Figuren der Comédie humaine des französischen Schriftstellers. Kommt ihn sein Bruder besuchen, so lesen beide gemeinsam ihren Balzac, den sie erstmals als Kinder in der Bibliothek ihres Vaters entdeckt hatten. Interessanterweise aber 
zitiert der Duc de Guermantes nicht nur am liebsten die unbekanntesten Werke Balzacs, sondern sehr gerne auch Werke von Autoren, die Zeitgenossen Balzacs waren, heute längst vergessen sind, die er aber in dasselbe aufwendige Kalbsleder hatte binden lassen, insofern sie eben derselben geschichtlichen Zeit angehörten. Welches aber sind nun die Konsequenzen hieraus? Lesen wir als Antwort eine Passage aus Contre Sainte-Beuve:

Wenn man die Bücher öffnete und dasselbe dünne Papier Ihnen in großen Schriftzeichen den Namen der Heldin präsentierte, so als wäre es absolut sie selbst, die sich Ihnen unter diesem tragbaren und komfortablen Aussehen vorstellte, begleitet von einem leichten Duft nach Klebstoff, von Staub und einem Alter, das wie die Ausstrahlung ihrer Reize war, dann fiel es einem recht schwer, zwischen diesen Büchern eine vorgeblich literarische Trennung einzuführen, welche künstlich auf Ideen beruhte, die ebenso dem Gegenstand des Romans wie dem Aussehen der Bände gänzlich fremd waren! ${ }^{7}$

Dieser kurze Einblick in die Privatbibliothek des Duc ist sehr aufschlussreich; zeigt er uns doch, wie sehr unser Lesen und unsere Aufnahme literarischer Werke von gänzlich äußerlichen Dingen wie dem aufwendigen Einband oder der Art des Klebstoffes, auf den ersten Blick völlig nebensächlichen materiellen Dingen, mitgesteuert werden. Ich möchte an dieser Stelle nicht auf die Rolle von Privatbibliotheken für die jeweiligen Gesellschaften und die Veränderungen der Lesarten und Leseweisen eingehen! Hier möge nur der Hinweis genügen, dass seit der römischen Kaiserzeit private Bibliotheken als Leseräume in zunehmendem Maße auch Lebensräume geworden waren; und ganz in diesem Sinne dürfen wir auch die Bibliothek des Duc de Guermantes verstehen, ist sie für ihn doch zugleich Zufluchtsort und Lebensort, an dem er auch bestimmte Lebensmittel zu sich zu nehmen pflegt ... seine aufmerksame Dienerschaft weiß ja Bescheid!

Doch es geht in dieser Passage zugleich um ein Zweites: Proust führt hier - anhand heute längst vergessener Autoren wie Roger de Beauvoir oder Céleste de Chabrillon - die haptische und olfaktorische Dimension des Buches ein, das gleichsam eine eigene Konkretheit in seiner Ausstattung und Lagerung erhält, welche stärker seien als jegliche literarische oder literarhistorische Trennung und Kategorisierung. Die Bücher werden gleichsam zu Angehörigen der selben Familie, auch wenn sie eigentlich von gänzlich verschiedenen Autoren stammen. Und ist es in der Tat nicht so, dass wir uns oft an die Ausstattung, an den Duft oder Geruch, an das Berühren eines bestimmten Papiertyps erinnern oder an die

7 Proust, Marcel: Contre Sainte-Beuve, suivi de Nouveaux Melanges, préface de Bernard de Fallois. Paris: Gallimard 1954, S. 237. 
Farben des Umschlages, während wir den Inhalt oder den Autornamen längst schon vergessen haben?

Die gleiche Ausstattung und die gemeinsame Lagerung gibt den Bänden eine fundamentale Gemeinsamkeit, die keineswegs nur visueller Natur ist, sondern alle Sinne anspricht und die Grenzen zwischen den Autorsubjekten sowie den Textinhalten verschwimmen lässt. So ist die Bibliothek nicht nur der Fluchtraum des Duc de Guermantes, in den er sich gerne bei einem Glas Sirup zurückzieht, sondern auch Ort einer ehemals bestehenden Beziehung zwischen Büchern von Autoren derselben Zeit, die in den Literaturgeschichten längst nicht mehr auftaucht, hier aber noch verstärkt wird dadurch, dass diese Bücher ihre eigene Zeitlichkeit und Räumlichkeit in eine gleichartige physische Körperlichkeit und Materialität umgewandelt haben; sie sind kaum mehr voneinander zu unterscheiden.

Die Erzählerfigur in Prousts Contre Sainte-Beuve versucht keineswegs, die Leseweise des Herzogs von Guermantes ins Lächerliche zu ziehen, sondern bezieht in ihre eigene Form des Lesens ebenfalls eine Vielzahl an lebensweltlichen und alltagsweltlichen Elementen und Details mit ein, die wir hier sicherlich einem zu erzielenden effet de réel zuordnen dürfen. Zugleich werden die Situationen des Lesens deutlicher und fassbarer konturiert. So heißt es denn auch wenig später von Seiten des Proust'schen Erzählers:

Und bisweilen frage ich mich, ob noch heute meine Art zu lesen nicht wesentlich stärker jener des Herrn von Guermantes ähnelt als jener der zeitgenössischen Kritiker. Ein Werk ist für mich noch immer ein lebendiges Ganzes, mit dem ich von der ersten Zeile an Bekanntschaft mache, dem ich mit Ehrerbietung zuhöre, dem ich Recht gebe, solange ich mit ihm zusammen bin, ohne eine Auswahl zu treffen und ohne zu diskutieren. ${ }^{8}$

Man könnte hier also von persönlichen ,Begegnungen mit Büchern‘ sprechen, die gegenüber der Begegnung mit Freunden oder der Begegnung im Rahmen der mondänen Konversation den enormen Vorteil der Schriftlichkeit besitzen. Aus unserer Perspektive entscheidend ist freilich der Hinweis darauf, dass ein Buch für den Proust'schen Erzähler ein lebendiges Ganzes ist, das heißt keineswegs etwas für immer Fixiertes, Festgestelltes, sondern eine lebendige und offene Struktur, die ein Lebenswissen nicht nur enthält, sondern auch lebendig und damit aktiv verkörpert und der Leserschaft anbietet.

Genau dies aber beinhaltet zugleich, dass das Lesen eine letztlich ergebnisoffene, weil ständig in Bewegung befindliche Aktivität ist; und da man Leben im Übrigen auch in den Naturwissenschaften nicht zuletzt dadurch definieren kann, dass es nicht in der Addition der Einzelteile aufgeht, sondern etwas mehr als

8 Proust, Marcel: Contre Sainte-Beuve, S. 238. 
die Summe dieser Fragmente ist, wird deutlich, dass jede Begegnung mit einem Buch und letztlich jedes Buch auch ein Eigenleben besitzt. Es verfügt über einen eigenen Raum, eine eigene Zeitlichkeit und schließlich auch ein eigenes Leben, das natürlich auch als Lebenswissen im Buch und als Buch mit seinem Lebenswissen gespeichert ist.

Das Buch hat im Dialog mit der Leserschaft immer recht; die Leserin und der Leser hören dem Buch zu und verleihen ihm Autorität. Das Lesen wird so $\mathrm{zu}$ einer keineswegs asymmetrischen Angelegenheit, bei der die Leserschaft gleichsam nach Belieben Gewalt über das Buch ausüben und bestimmte Passagen vorziehen, andere verdammen könnte. Vielmehr steht das Buch mit seinem Eigenleben im Mittelpunkt und wird als Buch zu einem Lebensmittelpunkt (und Lebensmittel) für den Erzähler selbst, dessen Lektüreweise sich damit jener des Duc de Guermants annähert. Dabei - dies bliebe noch hinzuzufügen - geht es bei Proust nie um ein einziges Buch, sondern um das Gesamtwerk eines Autors und damit um ein vielstimmiges Konzert. Auch hierin aber ist Proust letztlich an einem lebendigen Gegenstand ausgerichtet, der dank des ihm eigenen Lebens die Initiative ergreifen, Überzeugungen aussprechen, in einen Dialog mit dem Lesepublikum treten kann und erst wieder in eine leblose Dingwelt zurückfällt, sobald der Akt des Lesens - und ich meine dies nicht nur im Iser'schen Sinne, sondern mit allen Konnotationen - zu Ende ist und verklingt. 